\title{
Double-Step Adaptation of Saccadic Eye Movements Is Influenced by Priming with Age Stereotypies
}

\author{
Otmar Bock $^{1}$, Valentina Grigorova ${ }^{2}$, Milena Ilieva ${ }^{2}$ \\ ${ }^{1}$ Institute of Physiology and Anatomy, German Sport University, Köln, Germany \\ ${ }^{2}$ Institute of Neurobiology, Bulgarian Academy of Sciences, Sofia, Bulgaria \\ Email: bock@dshs-koeln.de
}

Received August $13^{\text {th }}, 2013$; revised September $16^{\text {th }}, 2013$; accepted October $12^{\text {th }}, 2013$

\begin{abstract}
Copyright (C) 2013 Otmar Bock et al. This is an open access article distributed under the Creative Commons Attribution License, which permits unrestricted use, distribution, and reproduction in any medium, provided the original work is properly cited. In accordance of the Creative Commons Attribution License all Copyrights (C) 2013 are reserved for SCIRP and the owner of the intellectual property Otmar Bock et al. All Copyright (C) 2013 are guarded by law and by SCIRP as a guardian.
\end{abstract}

\begin{abstract}
Age related deficits of sensorimotor adaptation have been observed earlier with arm, but not with eye movements. Here we evaluate whether deficits of eye adaptation may depend on the subjects' believes about their own sensorimotor abilities. To find out, elderly subjects were primed with positive or negative age stereotypes using the scrambled-sentence task, and were then exposed to a double-step saccade adaptation task. The outcome was compared to data from an earlier study with unprimed elderly persons. We found adaptation to be stronger after positive priming than after negative or no priming, with no difference between the latter two. Aftereffects of adaptation were not modified by priming. From this we conclude that positive primes enhanced workaround strategies, but not adaptive recalibration, while negative primes failed completely, possibly because of a floor effect.
\end{abstract}

Keywords: Old Age; Plasticity; Strategies; Recalibration; Societal Stereotypies

\section{Introduction}

The human sensorimotor system adapts readily to a wide range of distortions (Bock, 2013): when the visual or the force environment is systematically altered, our behavior is initially perturbed but gradually normalizes with extended practice. This ability deteriorates in old age; however, it has been shown that the speed and magnitude of adaptive change are reduced, while aftereffects remain intact (Bock, 2005; Fernández-Ruiz, Hall, Vergara, \& Díaz, 2000; McNay \& Willingham, 1998). Since aftereffects are thought to reflect adaptive recalibration only, unbiased by strategies (McNay \& Willingham, 1998; Redding $\&$ Wallace, 1996), the above work indicates that strategic control but not adaptive recalibration is degraded in old age. Accordingly, age-related deficits of adaptive change were found to be associated with cognitive decline (Bock \& Girgenrath, 2006).

The above studies dealt with the effects of old age on the adaptation of arm movements. We have recently expanded this work to oculomotor saccades and found no age-related deficits for saccadic adaptation under single-task conditions, nor under dual-task conditions when the second task was spatially adjacent; an adaptation deficit only emerged when the second task was located further away (Bock et al., under review). We attributed that deficit to the well-known shrinkage of the attention focus in old age (Ball \& Owsley, 1993; Beurskens \& Bock, 2012; Sekuler \& Ball, 1986), and concluded that saccadic adaptation per se is largely age-independent.

It seems surprising that saccadic adaptation should be immune to age-related decay, while other characteristics of ocular saccades deteriorate: their speed and accuracy decreases (Bono et al., 1996; Paquette \& Fung, 2011), and their path across a visual scene becomes less efficient (Chapman \& Hollands, 2006; Maltz \& Shinar, 1999). In fact, elderly subjects in our preceding study didn't adapt as well as young ones (Bock et al., under review), only the deficit didn't reach statistical significance. With these arguments casting doubt on our earlier conclusion, we decided to scrutinize it with a fresh approach.

Following the arguments of others, we reasoned that sensorimotor performance in old age is determined not only by biological decay, but also by the actor's confidence in her/his own capacity. This has been shown by studies which manipulated the self-confidence of seniors by priming them with societal preconceptions about the abilities of elderly persons: positive age stereotypes (words such as "mature") enhanced, and negative ones (words such as "frail") degraded seniors' performance on tasks such as locomotion (Hausdorff, Levy, \& Wei, 1999), chair rising (Levy, 2000) and handwriting (Levy \& Leifheit-Limson, 2009). We hypothesized that saccadic adaptation is affected by self-confidence as well, and therefore is impaired in elderly persons who assimilate negative societal stereotypes towards old age, but not in those who assimilate positive ones. This differential compliance with societal stereotypes would increase intersubject variability, and thus might have reduced the significance level for age differences in our preceding adaptation study to below 0.05 . To explore this view, we now compare saccadic adaptation in elderly participants primed with positive versus negative stereotypes of old age.

\section{Methods}

Sixteen healthy right-handed subjects participated. They 
were $58.7 \pm 4.4$ year old, had normal or corrected- to- normal vision, were native Bulgarian speakers with different education levels, and were naïve to the purposes of the experiment. All signed an informed consent to this study, which was pre-approved by the Ethics Committee of the Institute of Neurobiology at the Bulgarian Academy of Sciences in Sofia.

Subjects were first primed with age stereotypes using an established procedure, the scrambled sentence task (Bargh, Chen, \& Burrows, 1996). Each person was given 20 lists of five words, and had to select four words from each list to formulate a meaningful sentence; the fifth, non-fitting word had to be crossed out. Unbeknown to the subjects, one of the four selected words was either a positive or a negative age stereotype (e.g., "wisdom" versus "decay"). Eight subjects received lists with positive stereotypes, and the other eight with negative ones; all were instructed to proceed at their own pace. The prime words came from a pilot study in which eight persons, aged 50 to 65 , rated a list of 15 experimenter-selected words on two ten-point Likert scales, one scale for "maturity" and the other for "frailty". The 10 words with the highest "maturity" rating (5.0 to 8.0) and the 10 with the highest "frailty" rating (6.3 to 8.3 ) were repeatedly used as primes for the main experiment.

The scrambled sentence task was followed by a saccade adaptation task, using procedures established in our earlier work (Bock, Schmitz, \& Grigorova, 2008; Grigorova, Bock, \& Borisova, 2013). In single-step trials, a target of $7 \mathrm{~mm}$ diameter was presented in the center of the screen for $750-1500 \mathrm{~ms}$, enclosed by a circle of $11 \mathrm{~cm}$ radius. The target then jumped onto the circle in one of eight randomly selected directions $(0$, 45, 90, $315 \mathrm{deg}$, where $0 \mathrm{deg}$ denotes rightwards, and $90 \mathrm{deg}$ upwards), and returned to the center $760 \mathrm{~ms}$ later. In double-step trials, the target jumped onto the circle, shifted along the circle by $-15 \mathrm{deg}$ (clockwise) after $200 \mathrm{~ms}$, and returned to the center $640 \mathrm{~ms}$ later. Twenty successive trials constituted an episode, and were separated from the next episode by a short rest break. The adaptation task consisted of a baseline phase with two single-step episodes, followed by an adaptation phase with 25 double-step episodes, and then by an aftereffect phase with two single-step episodes.

The data of eight subjects from another study (Bock et al., under review) were used as non-priming control. Those subjects participated in the saccade adaptation task but not in the scrambled sentence task, were healthy, right-handed and $58.7 \pm$ 4.4 years old.

Horizontal and vertical eye movements were registered by electrooculography (DC-EOG) with a band pass filter of 0.08 $100 \mathrm{~Hz}$. Signals were digitized with a resolution of $0.01 \mathrm{deg} / \mathrm{bit}$ and a sampling rate of $100 \mathrm{~Hz}$, and calibration was repeated every five episodes. Custom-designed interactive software determined saccade direction as the angular difference between first target step and primary saccade, in the plane of the screen. The software also determined saccade latency as the delay between first target step and the onset of primary saccade. Saccades with latencies beyond $270 \mathrm{~ms}$ were discarded, since they may be influenced by reprogramming towards the second step (Becker \& Jürgens, 1979). Mean saccade directions for each subject and episode were adjusted by subtracting the subject-specific baseline values before graphical presentation and statistical analysis.

\section{Results}

Figure 1 illustrates that during the adaptation phase, saccade

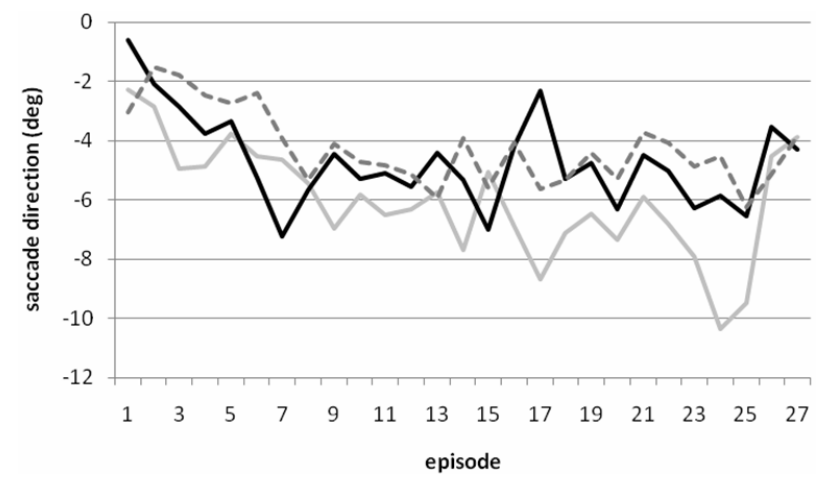

Figure 1.

Saccade direction for each episode of the adaptation phase (25 episodes) and the aftereffect phase ( 2 episodes). Means across subjects primed with positive age stereotypes are shown in grey, those across subjects primed with negative age stereotypes in black, and those across nonprimed control subjects are dashed. For better clarity, no error bars are shown.

direction gradually increased towards the negative, as expected for adequate adaptation. This increase was most pronounced in the group primed with positive age stereotypes, reaching a mean of $-8.1 \mathrm{deg}$ across the last five adaptation episodes. The five-episode mean of saccade direction was only $-4.7 \mathrm{deg}$ in the control group and $-4.4 \mathrm{deg}$ in the negatively primed group. When a deviant, non-adapting subject was excluded $(84 \%$ of data from that subject were positive rather than negative), the five-episode mean in the negatively primed group changed to -5.6 deg. In fact, the deviant subject is excluded in Figure 1 and in the reported statistical analyses. The five-episode means of saccade direction in all subjects were submitted to an one-way analysis of variance (ANOVA) with the factor Group, which yielded statistical significance $(\mathrm{F}(2,20)=9.38 ; p=$ $0.0013)$. Fisher's LSD post-hoc tests revealed significant differences between positive and negative group $(p<0.01)$, between positive and control group $(p<0.001)$ but not between negative and control group $(p>0.05)$.

Figure 1 further shows that saccade direction during the last two (aftereffect) episodes was quite similar in all groups. Accordingly, when mean saccadic direction across both aftereffect episodes was submitted to one-way ANOVA, no significance was yielded $(\mathrm{F}(2,20)=0.014 ; p>0.05)$.

\section{Discussion}

The present study manipulated subjects' performance through semantic priming. This technique is thought to activate a specific node in a lexical network and thus to facilitate the subsequent processing of words with similar meaning (Kiesel, Kunde, \& Hoffmann, 2007). However, it also was found to influence higher-order mental functions such as creativity (Mayer \& Mussweiler, 2011), self-confidence (Levy, Hausdorff, Hencke, \& Wei, 2000), product preference (Strahan, Spencer, \& Zanna, 2002) attention focus (Hüttermann, Memmert, \& Bock, 2012) and motivation (Hart \& Albarracín, 2009; Radel, Sarrazin, Legrain, \& Gobancé, 2009). We used this approach to activate positive or negative age stereotypes in our subjects, and observe the consequences on saccadic adaptation.

Our data document a beneficial influence of positive primes on performance during the adaptation phase, but not during the aftereffect phase. This suggests, according to established rea- 
soning (Bock, 2005; McNay \& Willingham, 1998), that positive age stereotypes enhanced workaround strategies but not adaptive recalibration. Somewhat surprisingly, negative primes had no noticeable effect at all: they modified neither recalibration nor strategies. One possible explanation is that priming with negative age stereotypes is less efficient than priming with positive ones. Another explanation could be social: maybe subjects came to the laboratory already with a negative attitude towards old age, and negative priming was therefore precluded by a floor effect. There indeed exists empirical evidence that in Bulgaria, compared e.g. to Germany, old age is regarded less favorably. Bulgarians rate the social status and the economical contribution of elderly persons much lower than Germans do, and rate their own experience of discrimination due to old age as much higher than Germans do (Abrams, Vauclair, \& Swift, 2011). These findings might be related to the fact that suicide rates among the elderly are substantially higher in Bulgaria than in Germany (Shah, Bhat, McKenzie, \& Koen, 2007). To decide between the two explanations, it would be desirable to compare the effects of positive versus negative age priming in different cultures, including those who traditionally venerate their elders. In this respect, it is interesting to note (Levy \& Langer, 1994) that memory loss in old age is more severe among Americans with intact hearing than in American Deaf (who don't experience spoken age stereotypes), and also more severe than in mainland Chinese with intact hearing (who live in a culture that honors old age).

The outcome of our study is in accordance with the hypothesis stated in the Introduction section. Thus, the strategic contribution towards saccadic adaptation might deteriorate in some seniors more than in others, depending on their assimilation of societal stereotypes, and only reaches statistical significance when the role of stereotypes is controlled for by priming. This could explain why no reliable deficit was observed when unprimed older subjects were compared to young ones (Bock et al., under review), but a robust deficit emerged when older negatively primed subjects were compared to older positively primed ones in the present study. In any case, age-related decrements of saccadic adaptation, like those of arm adaptation (Bock, 2005; Fernández-Ruiz et al., 2000; McNay \& Willingham, 1998), seem to afflict strategies but not recalibration. The decrements seem to be less pronounced for the eyes than for the arm, where a significant difference between age groups was repeatedly observed even without primes (Bock, 2005; Fernández-Ruiz et al., 2000; McNay \& Willingham, 1998), possibly because of a stronger cortical involvement in the control of arm versus eye movements.

\section{Conclusion}

The present study takes its place alongside other researches that documented the role of social preconceptions on sensorimotor performance (Hausdorff et al., 1999; Levy, 2000; Levy \& Leifheit-Limson, 2009), and thus highlights the potential dangers of a vicious circle where negative attitudes towards old age may activate performance deficits in the elderly by way of self-fulfilling prophecy, which in turn enforces the negative attitudes.

\section{REFERENCES}

Abrams, D., Vauclair, C.-M., \& Swift, H. (2011). Predictors of attitudes to age across Europe. London: Department for Work and Pen- sions.

Ball, K., \& Owsley, C. (1993). The useful field of view test: A new technique for evaluating age-related declines in visual function. Journal of the American Optometric Association, 64, 71-79.

Bargh, J. A., Chen, M., \& Burrows, L. (1996). Automaticity of social behavior: Direct effects of trait construct and stereotype-activation on action. Journal of Personality and Social Psychology, 71, 230244. http://dx.doi.org/10.1037/0022-3514.71.2.230

Becker, W., \& Jürgens, R. (1979). An analysis of the saccadic system by means of double step stimuli. Vision Research, 19, 967-983. http://dx.doi.org/10.1016/0042-6989(79)90222-0

Beurskens, R., \& Bock, O. (2012). Age-related decline of peripheral visual processing: The role of eye movements. Experimental Brain Research, 217, 117-124.

http://dx.doi.org/10.1007/s00221-011-2978-3

Bock, O. (2005). Components of sensorimotor adaptation in young and elderly subjects. Experimental Brain Research, 160, 259-263. http://dx.doi.org/10.1007/s00221-004-2133-5

Bock, O. (2013). Basic principles of sensorimotor adaptation to different distortions with different effectors and movement types: A review and synthesis of behavioral findings. Frontiers Hum Neuroscience, 7, 81. http://dx.doi.org/10.3389/fnhum.2013.00081

Bock, O., \& Girgenrath, M. (2006). Relationship between sensorimotor adaptation and cognitive functions in younger and older subjects. Experimental Brain Research, 169, 400-406. http://dx.doi.org/10.1007/s00221-005-0153-4

Bock, O., Schmitz, G., \& Grigorova, V. (2008). Transfer of adaptation between ocular saccades and arm movements. Human Movement Science, 27, 383-395. http://dx.doi.org/10.1016/j.humov.2008.01.001

Bono, F., Oliveri, R., Zappia, M., Aguglia, U., Puccio, G., \& Quattrone, A. (1996). Computerized analysis of eye movements as a function of age. Archives of Gerontology and Geriatrics, 22, 261-269. http://dx.doi.org/10.1016/0167-4943(96)00698-X

Chapman, G. J., \& Hollands, M. A. (2006). Evidence for a link between changes to gaze behaviour and risk of falling in older adults during adaptive locomotion. Gait \& Posture, 24, 288-294.

http://dx.doi.org/10.1016/j.gaitpost.2005.10.002

Fernández-Ruiz, J., Hall, C., Vergara, P., \& Díaz, R. (2000). Prism adaptation in normal aging: Slower adaptation rate and larger aftereffect. Cognitive Brain Research, 9, 223-226. http://dx.doi.org/10.1016/S0926-6410(99)00057-9

Grigorova, V., Bock, O., \& Borisova, S. (2013). Concurrent adaptation of reactive saccades and hand pointing movements to equal and to opposite changes of target direction. Experimental Brain Research, 226, 63-71. http://dx.doi.org/10.1007/s00221-013-3411-x

Hart, W., \& Albarracín, D. (2009). The effects of chronic achievement motivation and achievement primes on the activation of achievement and fun goals. Journal of Personality and Social Psychology, 97, 1129-1141. http://dx.doi.org/10.1037/a0017146

Hausdorff, J. M., Levy, B. R., \& Wei, J. Y. (1999). The power of ageism on physical functions of older persons: Reversibility of age-related gait changes. Journal of the American Geriatrics Society, 47, 13461349.

Hüttermann, S., Memmert, D., \& Bock, O. (2012). Semantic priming of attention focus: Evidence for short- and long-term effects. Psychology, 3, 128-131. http://dx.doi.org/10.4236/psych.2012.32019

Kiesel, A., Kunde, W., \& Hoffmann, J. (2007). Mechanisms of subliminal response priming. Advanced Cognitive Psychology, 3, $307-$ 315. http://dx.doi.org/10.2478/v10053-008-0032-1

Levy, B. (2000). Handwriting as a reflection of aging self-stereotypes. Journal of Geriatric Psychiatry, 33, 81-94.

Levy, B. R., Hausdorff, J. M., Hencke, R., \& Wei, J. Y. (2000). Reducing cardiovascular stress with positive self-stereotypes of aging. The Journals of Gerontology: Series B, 55, 205-213. http://dx.doi.org/10.1093/geronb/55.4.P205

Levy, B. R., \& Leifheit-Limson, E. (2009). The stereotype-matching effect: Greater influence on functioning when age stereotypes correspond to outcomes. Psychology and Aging, 24, 230-233. http://dx.doi.org/10.1037/a0014563

Levy, B., \& Langer, E. (1994). Aging free from negative stereotypes: successful memory in China and among the American deaf. Journal 
of Personality and Social Psychology, 66, 989-997.

http://dx.doi.org/10.1037/0022-3514.66.6.989

Maltz, M., \& Shinar, D. (1999). Eye movements of younger and older drivers. Human Factors, 41, 15-25.

http://dx.doi.org/10.1518/001872099779577282

Mayer, J., \& Mussweiler, T. (2011). Suspicious spirits, flexible minds: When distrust enhances creativity. Journal of Personality and Social Psychology, 101, 1262-1277. http://dx.doi.org/10.1037/a0024407

McNay, E., \& Willingham, D. (1998). Deficit in learning of a motor skill requiring strategy, but not of perceptualmotor recalibration, with aging. Learning Memory, 4, 411-420.

http://dx.doi.org/10.1101/1m.4.5.411

Paquette, C., \& Fung, J. (2011). Old age affects gaze and postural coordination. Gait \& Posture, 33, 227-232.

http://dx.doi.org/10.1016/j.gaitpost.2010.11.010

Radel, R., Sarrazin, P., Legrain, P., \& Gobancé, L. (2009). Subliminal priming of motivational orientation in educational settings: Effect on academic performance moderated by mindfulness. Journal of Re- search in Personality, 43, 695-698.

http://dx.doi.org/10.1016/j.jrp.2009.02.011

Redding, G. M., \& Wallace, B. (1996). Adaptive spatial alignment and strategic perceptual-motor control. Journal of Experimental Psychology: Human Perception and Performance, 22, 379-394. http://dx.doi.org/10.1037/0096-1523.22.2.379

Sekuler, R., \& Ball, K. (1986). Visual localization: Age and practice. Journal of the Optical Society of America A, 3, 864-867. http://dx.doi.org/10.1364/JOSAA.3.000864

Shah, A., Bhat, R., McKenzie, S., \& Koen, C. (2007). Elderly suicide rates: Cross-national comparisons and association with sex and elderly age-bands. Medicine, Science and the Law, 47, 244-252.

http://dx.doi.org/10.1258/rsmmsl.47.3.244

Strahan, E., Spencer, S. J., \& Zanna, M. P. (2002). Subliminal priming and persuasion: Striking while the iron is hot. Journal of Experimental Social Psychology, 38, 556-568.

http://dx.doi.org/10.1016/S0022-1031(02)00502-4 